ENVIRONMENTAL EXPOSURE

\title{
Association between short term exposure to fine particulate matter and heart rate variability in older subjects with and without heart disease
}

\author{
J H Sullivan, A B Schreuder, C A Trenga, S L-J Liu, T V Larson, J Q Koenig, J D Kaufman
}

Thorax 2005;60:462-466. doi: 10.1136/thx.2004.027532

\begin{abstract}
See end of article for authors' affiliations

Correspondence to: Dr J H Sullivan, Northwest Particulate Matter Center, Suite 355, $1107 \mathrm{NE} 45$ th Street, Seattle 98105, USA;

sullih@u.washington.edu
\end{abstract}

Received 29 April 2004

Accepted 5 January 2005
Background: Short term increases in exposure to particulate matter (PM) air pollution are associated with increased cardiovascular morbidity and mortality. The mechanism behind this effect is unclear, although changes in autonomic control have been observed. It was hypothesised that increases in fine PM measured at the subjects' home in the preceding hour would be associated with decreased high frequency heart rate variability (HF-HRV) in individuals with pre-existing cardiac disease.

Methods: Two hundred and eighty five daily 20 minute measures of HRV (including a paced breathing protocol) were made in the homes of 34 elderly individuals with $(n=21)$ and without $(n=13)$ cardiovascular disease (CVD) over a 10 day period in Seattle between February 2000 and March 2002. Fine PM was continuously measured by nephelometry at the individuals' homes.

Results: The median age of the study population was 77 years (range 57-87) and $44 \%$ were male. Models that adjusted for health status, relative humidity, temperature, mean heart rate, and medication use did not find a significant association between a $10 \mu \mathrm{g} / \mathrm{m}^{3}$ increase in 1 hour mean outdoor PM 2.5 before the HRV measurement and a change in HF-HRV power in individuals with CVD $13 \%$ increase in median HF-HRV (95\% Cl -19 to 32$)$ ) or without CVD (5\% decrease in median HF-HRV (95\% Cl -34 to 36$)$ ). Similarly, no association was evident using 4 hour and 24 hour mean outdoor $\mathrm{PM}_{2.5}$ exposures before the HRV measurement.

Conclusion: No association was found between increased residence levels of fine PM and frequency domain measures of HRV in elderly individuals.
(1)= ncreased levels of particulate matter air pollution (PM) have been associated with increased cardiovascular morbidity and mortality in elderly individuals with pre-existing cardiac and pulmonary disease. ${ }^{1-4}$ Furthermore, studies have shown that PM is associated with increased automatic implantable cardioverter defribillator (AICD) discharges ${ }^{5}$ and may trigger the onset of myocardial infarction (MI), suggesting a potential pro-arrhythmic effect.

Despite substantial in vitro, in vivo, and human epidemiological investigations, the potential mechanism of the effect of PM on the cardiovascular system remains unknown. One potential mechanism is an altered autonomic regulation of cardiac rhythm. ${ }^{78}$ Animal and human electrophysiological studies suggest that changes in the autonomic regulation of the heart may contribute to the occurrence of ventricular arrhythmias. ${ }^{9-11}$ Cross sectional studies in individuals with and without pre-existing heart disease show that a baseline measure of decreased heart rate variability (HRV), a noninvasive measure of autonomic control of the heart, predicts an increased risk of developing MI and sudden death. ${ }^{12-15}$ Moreover, PM has been associated with a decline in HRV in previous air pollution panel studies and a large cross sectional community study. ${ }^{16-22}$ However, these panel studies have relied primarily on central site exposure measures and included small numbers of individuals with pre-existing cardiac disease. Even with these limitations, they suggest a role for decreased parasympathetic modulation of HRV by increased ambient derived PM exposures.

To expand on this earlier work, we have performed a study in Seattle pairing daily measures of HRV with continuous short term mean fine PM measurements at the homes of elderly individuals with and without pre-existing cardiac disease. We hypothesised that increased short term exposure to fine PM would result in decreased high frequency (HF) HRV, especially in individuals with pre-existing cardiac disease. We further hypothesised that PM measured outside the subjects' homes would have a greater effect than indoor PM.

\section{METHODS}

Daily 20 minute, supine, three-channel continuous Holter monitor measurements (Del Mar, Irvine, CA, USA) were performed in the homes of 34 elderly individuals with and without CVD during a 10 day monitoring period between February 2000 and March 2002. Twenty five individuals were monitored over a single 10 day session and nine individuals were monitored over two $(n=8)$ or three sessions $(n=1)$. These data were paired with indoor and outdoor nephelometry measurements of fine PM made at the individuals' homes. These data were further enhanced by meteorological variables: hourly means of relative humidity (RH) and temperature from a central site air pollution monitor (Puget Sound Clean Air Agency); daily questionnaire responses on medication use, cardiac symptoms and clinic visits; and physiological measurements of sitting blood pressure, pulse rate, and oxygen saturation prior to HRV measurements.

The study protocol was approved by the University of Washington institutional review board.

\section{Study population}

To assess eligibility, each potential subject had a baseline ECG to exclude cardiac arrhythmias or conduction

Abbreviations: $A I C D$, automatic implantable cardioverter defribillator; $\mathrm{CVD}$, cardiovascular disease; HRV, heart rate variability; MI, myocardial infarction; PM, particulate matter 
disturbances. To optimise interpretation of HRV measurements we excluded individuals with atrial fibrillation, second degree heart block, left bundle branch block, paced rhythm, or known diabetes mellitus. Subjects were also excluded if they had unstable angina, MI, pneumonia, or an exacerbation of chronic obstructive pulmonary disease within 30 days of the study. No subject had a known MI within 18 months of the study.

\section{HRV measurements}

HRV was measured at the individual's residence at the same time of day by trained study technicians. The measurements were performed after the subjects had rested in the supine position for 10 minutes. To diminish the effect of respiratory variation on HRV, participants followed a paced breathing protocol using audio cues at a respiratory rate of 12 . The electrodes were placed in a modified V1-V5 position.

\section{Processing of HRV measurements}

A trained study technician reviewed and edited automatically determined readings of QRS complexes. Regions of artifact were eliminated. No recordings with more than $1 \%$ ectopic beats or 5\% artifact were considered for analysis. After correction the cardiac rhythm data were analysed using Del Mar Dartscan software (Model DS-90, Irvine, CA, USA). Only normal-normal intervals between normal sinus rhythms with a successive $\mathrm{N}-\mathrm{N}$ ratio of $0.8-1.2$ were included for HRV analysis. The standard analysis included both time domain (SDNN, SDANN, r-MSSD) and frequency spectral measures (total power, high frequency (HF, 0.15-0.4 Hz), low frequency (LF, 0.04-0.15 Hz), and very low frequency (VLF, $0.01-0.04 \mathrm{~Hz}$ ) HRV). ${ }^{23}$ The frequency measurement was obtained by performing a fast Fourier transformation method on 256 beat blocks sampled at 5 minute intervals using a Hamming windowed signal.

\section{Exposure measures}

The primary exposure metric was short term averaged $(1,4$, and 24 hour averaged) fine PM measured by nephelometry (Radiance Research, Seattle, WA, USA) as a light scattering coefficient outside the participant's residence. To allow for comparability with other studies we calibrated the nephelometric measure of PM against a gravimetric $\mathrm{PM}_{2.5}$ measure. This measure is referred to here as fine PM. Nephelometric data correlate well with gravimetric particle measurements in the $0.1-1.4$ aerodynamic range. ${ }^{24}$ We also measured 1,4 , and 24 hour mean indoor fine PM by nephelometry at the subject's residence. Outdoor nephelometry data were not measured for six individuals so we used the closest home outdoor nephelometer which was sited within 2 miles of the residences to capture local PM levels. A total of 28 days surrogate nephelometry exposure were used in our final analysis.

\section{Statistical analysis}

The data were analysed using SAS (Version 8.0, Cary, NC, USA). HRV power measures were log transformed before analyses.

A linear mixed model with random intercepts was used to determine the within individual effect of 1, 4, and 24 hour averaged outdoor PM on log transformed HF-HRV measures for individuals with and without heart disease. The final models were adjusted for age, mean resting heart rate, medication use, and meteorological variables (temperature and relative humidity as both linear and quadratic terms). The same models were repeated using indoor measures of fine PM. Individuals with COPD and healthy subjects were grouped as a non-CVD subgroup because of the small numbers.
Table 1 Demographic data of study participants

\begin{tabular}{|c|c|c|}
\hline Variable & $\begin{array}{l}\text { CVD } \\
(n=21)\end{array}$ & $\begin{array}{l}\text { No CVD } \\
(n=13)\end{array}$ \\
\hline Median (range) age (years) & $77(57-85)$ & 78 (72-87) \\
\hline Sex (male) & $9(43 \%)$ & $6(46 \%)$ \\
\hline Race (white) & $20(95 \%)$ & $12(92 \%)$ \\
\hline History of MI & $9(43 \%)$ & $0(0 \%)$ \\
\hline Current angina & $6(29 \%)$ & $0(0 \%)$ \\
\hline Current CHF & $3(14 \%)$ & $0(0 \%)$ \\
\hline Current hypertension & $9(43 \%)$ & $6(46 \%)$ \\
\hline \multicolumn{3}{|l|}{ Medication use ${ }^{*} \dagger$} \\
\hline$\beta$ blockers & $12(57 \%)$ & $0(0 \%)$ \\
\hline ACE inhibitors & $9(43 \%)$ & $4(31 \%)$ \\
\hline Calcium channel inhibitors & $2(10 \%)$ & $2(15 \%)$ \\
\hline \multicolumn{3}{|c|}{$\begin{array}{l}\text { CVD, cardiovascular disease; MI, myocardial infarction; CHF, congestive } \\
\text { heart failure; } \mathrm{ACE} \text {, angiotensin converting enzyme. } \\
\text { *Diuretics (furosemide, hydrochlorothiazide) were the most common anti- } \\
\text { hypertensive medication prescribed in the non-CVD population. } \\
\text { tMedication use adds up to more than } 100 \% \text { of the cardiac population as } \\
\text { some individuals were on more than one class of medications. }\end{array}$} \\
\hline
\end{tabular}

To further explore whether autonomic imbalance was responsible for the PM effect, we similarly modelled the effect of 1, 4, and 24 hour mean outdoor and indoor nephelometry measures of PM on r-MSSD, SDNN, LF, and the LF:HF ratio of HRV.

\section{RESULTS}

HRV measurements were performed in individuals with and without CVD over 2310 day study sessions. After editing, 285 HRV measurements were available in 34 individuals with $(\mathrm{n}=21)$ and without $(\mathrm{n}=13)$ CVD. After allowance for missing exposure data or unacceptable HRV measurements, a mean of six HRV measurements per individual was available for analysis in each study session.

The overall study population was older (median age 77 years (range 57-87)), predominantly white (94\%), and $44 \%$ were male (table 1 ). The demographic factors of the CVD and non-CVD study populations did not differ. The CVD population comprised three individuals with $\mathrm{CHF}$ and 18 with documented ischaemic heart disease without overt CHF. Beta-blockers were used in $57 \%$ of individuals with heart disease. Fifteen individuals were on antihypertensive medications, nine in the CVD group and six in the non-CVD group.

Over the 23 study sessions there was a modest range of within session 1 hour averaged PM exposure (median $10.6 \mu \mathrm{g} / \mathrm{m}^{3}$, range $3.1-40.4$; table 2). However, there was a relatively small range of 1 hour averaged exposure within individuals during each session (median $6.9 \mu \mathrm{g} / \mathrm{m}^{3}$, range 1.1-33.0; fig 1). Only 13 individuals ( six in the CVD group and seven in the non-CVD group) experienced an intrasession variation in 1 hour averaged $\mathrm{PM}_{2.5}$ exposure of more than $15 \mu \mathrm{g} / \mathrm{m}^{3}$.

Table 3 shows the range of HRV measurements in those with and without CVD. There was a slightly lower ln HF-HRV power in those with CVD $\left(5.0(1.2) \mathrm{ms}^{2}\right)$ than in those without CVD (5.6 (1.1) $\left.\mathrm{ms}^{2}\right)$. The range of measurements is consistent with previous studies of HRV in elderly populations. ${ }^{16} 19$

Final models adjusted for health status, relative humidity, temperature, and medication use did not find a significant association between a $10 \mu \mathrm{g} / \mathrm{m}^{3}$ increase in 1 hour mean outdoor $\mathrm{PM}_{2.5}$ preceding the HRV measurement and HF-HRV power in individuals with CVD ( $3 \%$ increase in median HFHRV (95\% CI -19 to 32 )) or those without CVD (5\% decrease in median HF-HRV (95\% CI -34 to 36 ), table 4 ). A similar null association was evident using 4 hour and 24 hour mean outdoor $\mathrm{PM}_{2.5}$ exposures before the HRV measurement 
Table 2 Summary of 1 hour mean outdoor exposure variables preceding HRV measurements

\begin{tabular}{lcccccc}
\hline Variable (unit) & Min & $\mathbf{2 5 \%}$ & $\mathbf{5 0 \%}$ & $\mathbf{7 5 \%}$ & $\mathbf{9 0 \%}$ & Max \\
\hline $\mathrm{PM}_{2.5}\left(\mu \mathrm{g} / \mathrm{m}^{3}\right) \dagger$ & 3.3 & 8.0 & 10.7 & 15.6 & 20.9 & 40.3 \\
$\mathrm{CO}(\mathrm{ppm})$ & 0.63 & 0.99 & 1.22 & 1.51 & 1.81 & 2.50 \\
$\mathrm{NO}_{2}(\mathrm{ppm})$ & 0.006 & 0.017 & 0.021 & 0.026 & 0.029 & 0.046 \\
Temperature & 33.5 & 40.9 & 46.4 & 53.3 & 61.3 & 69.3 \\
Relative humidity & 45.1 & 73.5 & 80.2 & 84.7 & 89.1 & 97.9 \\
\hline
\end{tabular}

†Represents $\mathrm{PM}_{2.5}$ equivalent of actual nephelometric measure.

$\mathrm{PM}_{2.5}=$ (nephelometry measure*100 $000-0.01$ ) $/ 0.28$.

The median and mean values for the 4 and 24 hour exposures are similar to the 1 hour mean values.

(table 4). Using the same model, no significant association was found for a $10 \mu \mathrm{g} / \mathrm{m}^{3}$ increase in 1 hour indoor measures of $\mathrm{PM}_{2.5}$ and HF-HRV power in those with CVD (13\% decrease in median HF-HRV (95\% CI -31 to 9$)$ ) or those without CVD (13\% increase in median HF-HRV (95\% CI - 17 to 53)). A similar null association was evident using 4 hour and 24 hour mean indoor $\mathrm{PM}_{2.5}$ exposures before the HRV measurement (data not shown). Similarly, analyses of secondary HRV end points (change in LF power and LF:HF ratio) in those with and without CVD showed no significant association (data not shown).

To confirm a genuine absence of autonomic influence of PM as suggested by our HF-HRV results, we performed similar analyses of the effect of a $10 \mu \mathrm{g} / \mathrm{m}^{3}$ increase in 1,4 , and 24 hour mean measures of outdoor and indoor $\mathrm{PM}_{2.5}$ on time domain indices of vagal control (r-MSSD). No significant association was found for a $10 \mu \mathrm{g} / \mathrm{m}^{3}$ increase in 1 hour mean outdoor $\mathrm{PM}_{2.5}$ preceding the HRV measurement and r-MSSD in individuals with CVD (4\% decrease in median r-MSSD (95\% CI -11 to 4$)$ ) or those without CVD (5\% increase in median r-MSSD [95\% CI -8 to 20), table 4). Similar null results were found for associations between 4 and 24 hour mean measures of outdoor $\mathrm{PM}_{2.5}$ and r-MSSD (table 4).

\section{DISCUSSION}

Our study did not find an association between an increase in the residence level of fine PM and frequency domain measurements of HRV in elderly individuals with and without CVD, in contrast to previous studies which have reported a relationship between ambient PM and decreased HRV. The absence of an association in our study may be because of the difference in composition of PM between study sites, low absolute levels and the small range in

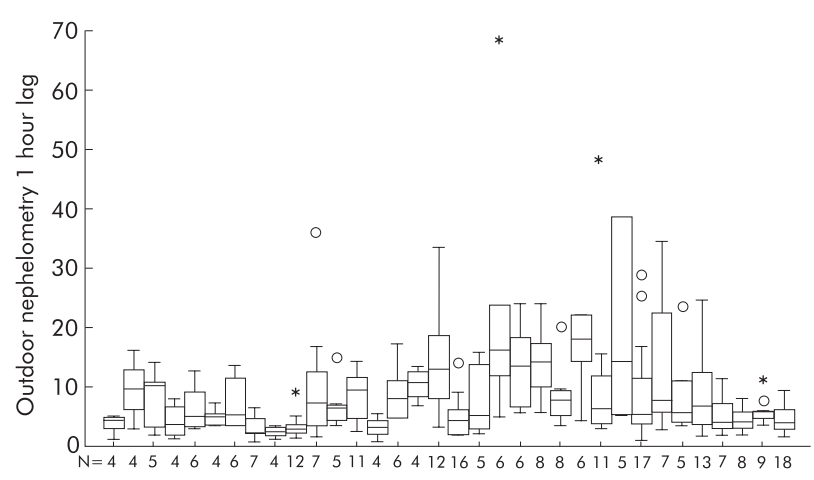

Figure 1 Summary of the within individual range of $\mathrm{PM}_{2.5}$ exposure over the study sessions. $\mathrm{N}$, number of exposure observations per individual during the study. Outdoor nephelometry converted to $\mathrm{PM}_{2.5}$ equivalent and expressed in $\mu \mathrm{g} / \mathrm{m}^{3}$. exposure variability during study sessions, dissimilarity in populations under study, or variation in the methods of HRV measurement.

Particulate matter in Boston and Baltimore is composed primarily of long range transport aerosols high in sulfate and trace metals. ${ }^{25}$ These particles may have a greater cardiovascular effect than combustion derived PM (wood smoke and vehicle emissions) which constitute the primary PM source in Seattle. Epidemiological studies have found a higher rate of cardiovascular mortality in the north eastern US (relative rate $0.6 \%$ ) than in the western US (relative rate $0.2 \%) .{ }^{26}$ Studies in the north eastern US have reported an association between raised PM levels and onset of $\mathrm{MI}^{5}$ and AICD firings; ${ }^{6}$ however, case crossover analyses in Seattle were unable to replicate these associations. ${ }^{27}{ }^{28}$ Furthermore, studies of hospital admissions for respiratory and cardiac diseases in eastern Canada suggest that PM with high sulfate composition has more deleterious cardiovascular effects than low sulfate $\mathrm{PM}_{2.5}{ }^{29}$ Moreover, in vitro and in vivo studies of PM stripped of the metal content found attenuation of both pulmonary and vascular inflammation ${ }^{30}$ and arrhythmogenic potential of $\mathrm{PM}_{2.5} \cdot{ }^{31}{ }^{32}$ Finally, a single study in healthy aluminium workers ${ }^{33}$ suggests that the metal composition of PM influences cardiac autonomic function, measured by HRV.

Alternatively, our inability to detect changes in the HRV may have been due to the small range of PM exposure (median IQR $6 \mu \mathrm{g} / \mathrm{m}^{3}$ ) over each individual's study session relative to the larger PM exposure range (median IQR 13$60 \mu \mathrm{g} / \mathrm{m}^{3}$ ) in previous studies which found a positive effect of PM on HRV measurements. ${ }^{16-1921}$ A threshold level of PM exposure may be required to induce a measurable change in autonomic control of the heart. Concentrated ambient particle exposures to $40.5(8.6) \mu \mathrm{g} / \mathrm{m}^{3} \mathrm{PM}_{2.5}$ performed in North Carolina on 10 healthy elderly human volunteers resulted in a decrease in HF-HRV immediately after exposure. $^{29}$ A recent investigation in a panel study of 88 elderly individuals in Utah by Pope estimated that a $100 \mu \mathrm{g} /$ $\mathrm{m}^{3}$ increase in 24 hour averaged $\mathrm{PM}_{2.5}$ measured at central monitors resulted in a mean (SE) decline in r-MSSD of 42 (11) ms when contrasting low and high PM days in the same individual. ${ }^{19}$ Their study participants experienced a higher PM exposure (mean (SD) $23.5(21) \mu \mathrm{g} / \mathrm{m}^{3}$ ) and the contrast in exposure between HRV measurements was much greater in these subjects, suggesting that their ability to detect an effect may be due to the greater exposure variability. Only six participants with CVD in our study experienced a variation in $\mathrm{HRV}$ as large as $15 \mu \mathrm{g} / \mathrm{m}^{3} \mathrm{PM}_{2.5}$ over one 10 day study session. Moreover, 14 individuals were exposed to PM levels below $10 \mu \mathrm{g} / \mathrm{m}^{3}$ during their entire study session.

To compare the findings of this study with previous studies of the effect of PM exposure on HRV requires understanding the differences in protocols and study populations between studies. ${ }^{16-22}$ Unlike Gold and Pope, we chose a frequency domain analysis with focus on HF power. HF-HRV spectral 
Table 3 Comparison of mean (SE) heart rate and heart rate variability (HRV) measurements according to the presence or absence of known cardiovascular disease (CVD)

\begin{tabular}{lllll}
\hline Variable & Non-CVD & CVD & Prior MI & Current CHF \\
\hline $\begin{array}{l}\text { Resting heart rate } \\
\text { (beats } / \mathrm{min})\end{array}$ & $72(10)$ & $72(12)$ & $70(9)$ & $75(8)$ \\
ln LF-HRV $\left(\mathrm{ms}^{2}\right)$ & $5.0(1.2)$ & $4.2(1.3)$ & $4.6(1.3)$ & $3.8(1.7)$ \\
In HF-HRV (ms $\left.{ }^{2}\right)$ & $5.6(1.1)$ & $5.0(1.2)$ & $5.1(1.2)$ & $4.9(1.2)$ \\
In RMSSD (ms) & $3.7(0.3)$ & $3.7(0.4)$ & $3.7(0.5)$ & $3.6(0.4)$ \\
In SDNN (ms) & $4.0(0.3)$ & $3.9(0.4)$ & $4.1(0.3)$ & $3.8(0.4)$ \\
\hline
\end{tabular}

analysis measurements may more accurately describe the autonomic contribution to cardiac oscillation than time domain measures in short term HRV recordings. ${ }^{23}$

Based on previous studies, we decided that a paced breathing protocol was essential to control for non-PM induced changes in vagal tone. We were unable to test whether the paced breathing protocol prevented small increases in the respiratory rate due to PM. Our results may differ as small changes in respiratory rate may have blunted the effect of PM on HF-HRV.

Panel studies by Liao $e t \mathrm{al}^{16}$ and Holguin et $\mathrm{al}^{21}$ found that PM caused a decline in HF-HRV measurements but these studies either excluded or sampled small numbers of individuals with pre-existing heart disease. Such subjects are considered more susceptible to the effects of fine PM. ${ }^{3} 47$ Moreover, studies have shown that individuals with preexisting cardiac disease have decreased baseline HF-HRV relative to healthy controls. ${ }^{13}{ }^{14}$ Within these populations, decreased baseline HF-HRV is predictive of future cardiac events. ${ }^{9}{ }^{13}{ }^{14}$ Lastly, animals with pre-existing cardiac disease have increased PM induced cardiac arrhythmia and decreased time domain measures of HRV compared with healthy controls. ${ }^{8}$

A recent large cross sectional study by Liao et a l $^{22}$ of 4899 elderly individuals found a decrease of $0.06 \mathrm{~ms}^{2}$ in HF-HRV for an $11 \mu \mathrm{g} / \mathrm{m}^{3}$ increase in $\mathrm{PM}_{10}$ air pollution, which suggests that increased 24 hour mean PM levels result in declines in HF-HRV. Interestingly, those with pre-existing cardiac disease were at greatest risk. The cross sectional nature of their study precluded determining temporal relations between PM and HRV in susceptible individuals. However, their large sample permitted detection of very small declines in HRV from ambient concentrations of PM. Despite their consistent findings of 24 hour mean levels of the effect

Table 4 Effect of a $10 \mu \mathrm{g} / \mathrm{m}^{3}$ increase in fine PM on HRV measurements in those with and without CVD adjusted for relative humidity, temperature, age, and medication use

\begin{tabular}{llll}
\hline $\begin{array}{l}\text { HRV } \\
\text { measurements }\end{array}$ & $\begin{array}{l}\text { Averaging } \\
\text { time }\end{array}$ & CVD & Non-CVD \\
\hline In HF-HRV & $1 \mathrm{~h}$ & $3(-19$ to 32$)$ & $-5(-34$ to 36$)$ \\
& $4 \mathrm{~h}$ & $8(-16$ to 38$)$ & $-7(-36$ to 34$)$ \\
& $24 \mathrm{~h}$ & $8(-19$ to 43$)$ & $-9(-40$ to 40$)$ \\
In r-MSSD & $1 \mathrm{~h}$ & $-4(-11$ to 4$)$ & $5(-8$ to 20$)$ \\
& $4 \mathrm{~h}$ & $-5(-12$ to 3$)$ & $7(-6$ to 23$)$ \\
& $24 \mathrm{~h}$ & $-5(-13$ to 3$)$ & $3(-11$ to 21$)$ \\
In SDNN & $1 \mathrm{~h}$ & $2(-4$ to 8$)$ & $-1(-15$ to 16$)$ \\
& $4 \mathrm{~h}$ & $3(-3$ to 9$)$ & $-1(-16$ to 16$)$ \\
& $24 \mathrm{~h}$ & $1(-5$ to 8$)$ & $1(-15$ to 22$)$ \\
\hline
\end{tabular}

Values are shown as percentage change from the median $(95 \% \mathrm{Cl})$ of PM on HRV, the small magnitude of the decline in HFHRV is not likely to account for the increased incidence of cardiac events on higher air pollution days. Finally, differences between the studies in the primary end point (baseline differences in HF-HRV in their population compared with within person change in HF-HRV in our study) and exposure measures (central site measures of PM compared with residential levels in our study) do not allow for a simple comparison of study findings.

In contrast to the study by Holguin et al, ${ }^{21}$ our study included individuals receiving $\beta$ blockers following MI. These drugs may have modulated the effect of PM on parasympathetic tone. A number of randomised clinical trials have found associations between the use of $\beta$ blockers after MI and an increase in HF-HRV. ${ }^{14} 34$

Potential strengths of our study include measures of HRV at the individual's residence, analysis of short term effects of PM on HRV, and inclusion of individuals with and without heart disease. The residential measurements of PM allowed for more precise assessment of exposure and a comparison between the indoor and outdoor effects of PM.

Potential weaknesses of our study include the small sample size and little variation in daily PM levels within sessions. However, we had more HRV measurements and a larger number of subjects than previous studies which found an association between PM and HRV.

Current air pollution studies assume that daily alterations in HRV are associated with an increased risk of arrhythmia. However, there are no studies relating short term alterations (daily, weekly) in HRV measures and clinical cardiac events. It is probably an oversimplification to consider a single noninvasive measure of cardiac function as an explanation or surrogate for the mechanism of the contribution of PM to excess cardiac mortality. Alterations in HRV may only be a contributing factor or a signal of susceptibility to the effects of PM. Previous studies of sudden cardiac death document many potential pathways leading to arrhythmia. ${ }^{35}$ Moreover, studies of alterations in HRV measures before AICD discharge in individuals with cardiomyopathy suggest that the low frequency measures of HRV are increased in the minutes before discharge..$^{36}$ This suggests a more complex balance of parasympathetic and sympathetic tone in modulating cardiac rhythm.

Our study was not able to detect a decrease in HRV in elderly individuals with and without known cardiac disease. However, we anticipate that future studies performed in larger populations with cardiac disease that combine multiple ECG measurements with systemic inflammatory and neurohormonal blood levels may detect potential pathways to account for the mechanism of the effect of PM in increasing mortality from cardiovascular disease. These studies should also be performed at a number of centres to determine whether the composition of PM influences the cardiac effect. 


\section{Authors' affiliations}

J H Sullivan, A B Schreuder, C A Trenga, S L-J Liu, T V Larson, J Q Koenig, J D Kaufman, Departments of Environmental and Occupational Health Sciences, Civil and Environmental Engineering, Biostatistics, and Medicine, University of Washington, Seattle, USA

The US Environmental Protection Agency through its Office of Research and Development partially funded and collaborated in the research described here under EPA grant R827355 to the University of Washington.

Mention of commercial products does not constitute an endorsement or recommendation for use.

\section{REFERENCES}

1 Dockery DW, Pope CA 3rd, Xu X, et al. $\mathrm{n}$ association between air pollution and mortality in six US cities. N Engl J Med 1993;329:1753-9.

2 Le Tertre A, Medina S, Samoli E, et al. Short-term effects of particulate air pollution on cardiovascular diseases in eight European cities. J Epidemiol Community Health 2002;56:773-9.

3 Samet JM, Dominici F, Curriero FC, et al. Fine particulate air pollution and mortality in 20 US cities, 1987-1994. N Engl J Med 2000:343:1742-9.

4 Schwartz J, Morris R. Air pollution and hospital admissions for cardiovascular disease in Detroit, Michigan. Am J Epidemiol 1995;142:23-35.

5 Peters A, Liu E, Verrier RL, et al. Air pollution and incidence of cardiac arrhythmia. Epidemiology 2000;11:11-7.

6 Peters A, Dockery DW, Muller JE, et al. Increased particulate air pollution and the triggering of myocardial infarction. Circulation 2001;103:2810-5.

7 Godleski JJ, Verrier RL, Koutrakis P, et al. Mechanisms of morbidity and mortality from exposure to ambient air particles. Res Rep Health Eff Inst 2000;5:88-103.

8 Cheng TJ, Hwang JS, Wang PY, et al. Effects of concentrated ambient particles on heart rate and blood pressure in pulmonary hypertensive rats. Environ Health Perspect 2003;111:147-50

9 Whitsel EA, Raghunathan TE, Pearce RM, et al. RR interval variation, the QT interval index and risk of primary cardiac arrest among patients without clinically recognized heart disease. Eur Heart J 2001;22:165-73.

10 Spooner PM, Albert C, Benjamin EJ, et al. Sudden cardiac death, genes, and arrhythmogenesis: consideration of new population and mechanistic approaches from a National Heart, Lung, and Blood Institute Workshop, Part l. Circulation $2001 ; 103: 2361-4$

11 Mehta MC, Jain AC, Mehta A, et al. Cardiac arrhythmias following intravenous nicotine: experimental study in dogs. $J$ Cardiovasc Pharmacol Ther 1997;2:291-8

12 Bigger JT Jr, Rolnitzky LM, Steinman RC, et al. Predicting mortality after myocardial infarction from the response of RR variability to antiarrhythmic drug therapy. J Am Coll Cardiol 1994;23:733-40.

13 Bilchick KC, Fetics B, Djoukeng R, et al. Prognostic value of heart rate variability in chronic congestive heart failure (Veterans Affairs' Survival Trial of Antiarrhythmic Therapy in Congestive Heart Failure). Am J Cardiol 2002;90:24-8

14 Hohnloser SH, Klingenheben T, Zabel M, et al. Heart rate variability used as an arrhythmia risk stratifier after myocardial infarction. Pacing Clin Electrophysiol 1997;20:2594-601.

15 La Rovere MT, Pinna GD, Maestri R, et al. Short-term heart rate variability strongly predicts sudden cardiac death in chronic heart failure patients. Circulation 2003;107:565-70.

16 Liao D, Creason J, Shy C, et al. Daily variation of particulate air pollution and poor cardiac autonomic control in the elderly. Environ Health Perspect 1999; 107:521-5.
17 Pope CA 3rd, Verrier RL, Lovett EG, et al. eart rate variability associated with particulate air pollution. Am Heart J 1999;138:890-9.

18 Gold DR, Litonjua A, Schwartz J, et al. Ambient pollution and heart rate variability. Circulation 2000;101:1267-73.

19 Pope CA 3rd, Hansen ML, Long RW, et al. mbient particulate air pollution, heart rate variability, and blood markers of inflammation in a panel of elderly subjects. Environ Health Perspect 2004;1 12:339-45.

20 Devlin RB, Ghio AJ, Kehrl H, et al. Elderly humans exposed to concentrated air pollution particles have decreased heart rate variability. Eur Respir J Suppl 2003:40:76-80s.

21 Holguin F, Tellez-Rojo MM, Hernandez M, et al. Air pollution and heart rate variability among the elderly in Mexico City. Epidemiology 2003;14:521-7.

22 Liao D, Duan Y, Whitsel EA, et al. Association of higher levels of ambient criteria pollutants with impaired cardiac autonomic control: a populationbased study. Am J Epidemiol 2004;159:768-77.

23 Task Force of the European Society of Cardiology and the North American Society of Pacing and Electrophysiology. Heart rate variability: standards of measurement, physiological interpretation and clinical use. Circulation 1996;93:1043-65.

24 Liu L-J, Slaughter JC, Larson TV. Comparison of light scattering devices and impactors for particulate measurements in indoor, outdoor, and personal environments. Environ Sci Technol 2002;36:2977-86.

25 Sarnat JA, Long CM, Koutrakis $P$, et al. Using sulfur as a tracer of outdoor fine particulate matter. Environ Sci Technol 2002;36:5305-14.

26 Samet JM, Zeger SL, Dominici F, et al. The National Morbidity, Mortality, and Air Pollution Study. Part II: Morbidity and mortality from air pollution in the United States. Res Rep Health Eff Inst 2000;94:5-79.

27 Sullivan J, Ishikawa N, Sheppard L, et al. Exposure to ambient fine particulate matter and primary cardiac arrest among persons with and without clinically recognized heart disease. Am J Epidemiol 2003;157:501-9.

28 Levy D, Sheppard L, Checkoway $H$, et al. A case-crossover analysis of particulate matter air pollution and out-of-hospital primary cardiac arrest Epidemiology 2001;12:193-9.

29 Goldberg MS, Burnett RT, Bailar JC. The association between daily mortality and ambient air particle pollution in Montreal, Quebec. 2. Cause-specific mortality. Environ Res $2001 ; 86: 26-36$.

30 Campen MJ, Nolan JP, Schladweiler MC, et al. Cardiovascular and thermoregulatory effects of inhaled PM-associated transition metals: a potential interaction between nickel and vanadium sulfate. Toxicol Sci 2001;64:243-52.

31 Molinelli AR, Madden MC, McGee JK, et al. Effect of metal removal on the toxicity of airborne particulate matter from the Utah Valley. Inhal Toxicol 2002;14:1069-86.

32 Magari SR, Schwartz J, Williams PL, et al. The association of particulate air metal concentrations with heart rate variability. Environ Health Perspect 2002; 110:875-80.

33 Welllenius G, Saldiva P, Batalha J, et al. Electrocardiographic changes during exposure to residual oil fly ash (ROFA) particles in a rat model of myocardial infarction. Toxicol Sci 2002;66:327-35.

34 Lampert R, Ickovics JR, Viscoli CJ, et al. Effects of propranolol on recovery of heart rate variability following acute myocardial infarction and relation to outcome in the Beta-Blocker Heart Attack Trial. Am J Cardiol 2003;91:137-42.

35 Priori SG, Aliot E, Blomstrom-Lundqvist C, et al. Task force on Sudden Cardiac Death of the European Society of Cardiology. Eur Heart $J$ 2001;22:1374-450.

36 Lombardi F, Porta A, Marzegalli $M$, et al. Heart rate variability patterns before ventricular tachycardia onset in patients with an implantable cardioverter defibrillator. Am J Cardiol 2000;86:959-63.

37 Pruvot E, Thonet G, Vesin JM, et al. Heart rate dynamics at the onset of ventricular tachyarrhythmias as retrieved from implantable cardioverterdefibrillators in patients with coronary artery disease. Circulation 2000; 101:2398-404 